\title{
Dietary Sodium and Blood Pressure in Older Adults
}

\author{
Courtney B. Johnson \\ University of Hawaii at Manoa, John A. Burns School of Medicine
}

\begin{abstract}
Given the proportion of older adults who are hypertensive and the population of older adults who are at risk for hypertension, the U.S. must mobilize public health efforts aimed at prevention. Scientific evidence has demonstrated the efficacy of sodium reduction to lower blood pressure. Translating this evidence into practice involves knowledge about the food sources of sodium so effective interventions can be designed and implemented. The purpose of this essay was to examine major food group sources of sodium in a cohort of older adults, with and without high blood pressure, in an urban community in Southwestern Pennsylvania. The University of Pittsburgh's "Center for Healthy Aging" promotes healthy aging in the community with the "10 Keys to Healthy Aging" campaign. One of the keys aims to lower systolic blood pressure to $\leq 140 \mathrm{mmHg}$. A low sodium intervention was implemented by the CHA project in hypertensive individuals. The sodium intake of the 521 community volunteers, mean age 74.5 years, $60 \%$ male, $94.1 \%$ white, who completed a FFQ, was compared to a sub-sample of hypertensives $(\mathrm{n}=214)$ who, in addition, collected one 24-hour urinary sodium. Mean baseline dietary sodium for the entire cohort was $1,796 \mathrm{mg}$ per day compared to $1,821 \mathrm{mg}$ per day in hypertensives. Urinary sodium was 1.8 times higher $(141 \mathrm{mmol} / 24 \mathrm{hrs}[3,240 \mathrm{mg}])$ than self-reported intake and decreased to $130 \mathrm{mmol} / 24 \mathrm{hrs}$ $(2,990 \mathrm{mg})$ at 6 -months. The correlation between dietary and urinary sodium at baseline was weak $(\mathrm{r}=0.16)$ and remained weak $(0.23)$ at 6-months. Major food sources of sodium were soups, breads, tomato sauce, salad dressings, and prepared cereals. Data indicate that the sodium intake of the group exceeds the 2005 Dietary Guidelines of $\leq 1500 \mathrm{mg}$ per day by approximately $200 \%$ for individuals at increased risk using urinary sodium values. Even the most successful dietary interventions to reduce sodium intake to the recommended levels would be ineffective without the food industry's help in reducing sodium added to foods during processing. This prevention strategy, in combination with stronger public health messages, would help to reduce the sodium intake in the population and help to achieve reductions in blood pressures.
\end{abstract}

(C) 2006 Californian Journal of Health Promotion. All rights reserved.

Keywords: hypertension, sodium, aging, prevention

\section{Introduction}

Evidence in older adults suggests that diets lower in sodium and higher in potassium and omega-3 fatty acids are probably beneficial in reducing the incidence of cardiovascular disease and stroke. Although the death rates for stroke and CHD have been declining over the past few decades, the population of older adults has increased and concurrently has the rates of CHD (Kochanek, Smith, Anderson, 2001). More than $40 \%$ of all deaths from heart disease and stroke can be accounted for from the 65-74 age group and subsequently increasing to nearly $60 \%$ in the 85 and older age groups (Kochanek, 2001). Many epidemiological studies identify several lifestyle and genetic factors that increase the risk of heart disease and stroke, which share many of the same risk factors. The World Health Organization (WHO) has estimated that high blood pressure causes approximately 1 in every 8 deaths worldwide, making hypertension the third leading cause of death in the world (WHO, 2002). Consequently, "Healthy People 2010" objectives (Table 1) (USDA, CDC, NIH, 2000) propose to decrease the proportion of adults with high blood pressure (HBP) to $16 \%$ from the current $28 \%$ by increasing the proportion of adults with controlled HBP and by improving the proportion of hypertensives on active therapy. 
Table 1

Healthy People 2010 Objectives Related to Hypertension

\begin{tabular}{|l|r|r|}
\hline \multicolumn{1}{|c|}{ Healthy People 2010 Objectives } & Baseline & Target \\
\hline Reduce coronary heart disease (CHD) deaths (per 100,000) & 208 & 166 \\
\hline Reduce stroke deaths (per 100,000) & 60 & 48 \\
\hline Reduce proportion of adults with high blood pressure & $28 \%$ & $16 \%$ \\
\hline $\begin{array}{l}\text { Increase proportion of adults with high blood pressure (HBP) whose blood } \\
\text { pressure is under control }\end{array}$ & $18 \%$ & $50 \%$ \\
\hline $\begin{array}{l}\text { Increase proportion of adults with HBP who are taking action (lifestyle } \\
\text { changes) to help control their blood pressure }\end{array}$ & $82 \%$ & $95 \%$ \\
\hline $\begin{array}{l}\text { Increase the proportion of physician office visits made by patients with a } \\
\text { diagnosis of cardiovascular disease, diabetes, or hyperlipidemia that } \\
\text { includes counseling or education related to diet and nutrition. }\end{array}$ & $42 \%$ \\
\hline $\begin{array}{l}\text { Increase proportion of older adults who have participated during the preceding } \\
\text { year in at least one organized health promotion activity. }\end{array}$ & $12 \%$ \\
\hline
\end{tabular}

Hypertension has been defined arbitrarily as a systolic blood pressure of $140 \mathrm{mmHg}$ or higher and a diastolic blood pressure of $90 \mathrm{mmHg}$ or higher, and includes individuals who are prescribed blood pressure medication(s) to control the condition. Although improving, only about one-third of Americans, ages 18-74, with hypertension are currently meeting this goal. Another one-third is still unaware of their hypertension and $40 \%$ of individuals with hypertension are not on treatment, according to the 1999-2000 NHANES data (Wright et al., 2003). Blood pressure control rates are only about $20 \%$ in older hypertensives, largely due to poor systolic control. More importantly, according to the 7th report of the Joint National Committee (JNC) on Detection, Evaluation, and Treatment of High Blood Pressure (JNC 7 Committee, 2003), cardiovascular risk from systolic hypertension begins at $115 \mathrm{mmHg}$ and risk from diastolic hypertension begins at 75 $\mathrm{mmHg}$, doubling with every $20 / 10 \mathrm{mmHg}$ (systolic/diastolic) increment. The first report of the JNC was published in 1977. This came after the National Heart, Lung and Blood Institute of the NIH recognized a need to organize a committee of representatives from most of the major domestic medical organizations. Since then reports have been issued approximately every 4 years, which serve as both consensus and evidence-based guidelines for the detection, treatment and evaluation of hypertension. The most recent report, JNC VII, was published in 2003 and focuses on the importance of systolic blood pressure, the value of prevention and the importance of lifestyle. It emphasizes that systolic blood pressure is a more important risk factor for cardiovascular disease (CVD) than diastolic blood pressure in adults over age 50 . Systolic blood pressure increases almost linearly with age, placing the entire adult population at risk (Figure 1). A normotensive individual at the age of 55 has a $90 \%$ lifetime risk of developing hypertension. At the same time, there is a three to four-fold increase in CVD risk in older compared to younger individuals (JNC 7 Committee, 2003).

To date, most dietary lifestyle approaches in adults have focused on altering key nutrients alone and in combination with weight loss as effective interventions to lower blood pressure in both men and women, all ages and in African Americans. Few studies have focused on the dietary patterns and important food group sources of key nutrients in older adults. Given the proportion of older adults who are hypertensive and the expanding population of older adults who are at risk for hypertension, the U.S. must mobilize public health efforts aimed at prevention. The purpose of this essay is to examine major food group sources of sodium in 
a cohort of older adults, with and without high blood pressure, in an urban community in Southwestern Pennsylvania (PA). Information about the dietary intake of these individuals will help advance the design of effective interventions to lower systolic blood pressures.

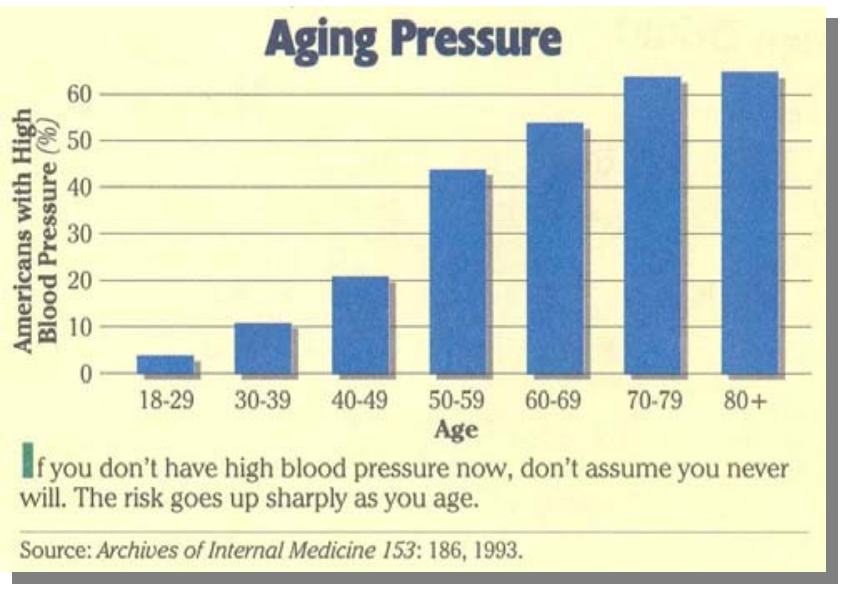

Figure 1

Affects of Age on Blood Pressure

As the population ages and since blood pressure increases with age, prevention and treatment across older adults is needed. Effective blood pressure control is achievable yet the majority of patients will likely require pharmacologic approaches in combination with progressive lifestyle interventions (JNC \& Committee, 2003). Although hypertension is more prevalent in older adults, recommendations are very similar. Due to co-morbidities and risk for elevated symptoms, drug treatment doses may be lower at initial treatment but this high-risk group may require multiple drugs to reach blood pressure targets (JNC 7 Committee, 2003). To reach the goal of reducing the public health burden of cardiovascular morbidity and mortality, the adoption of healthy lifestyle interventions is key not only for the management of high blood pressure, but more importantly for prevention. Nonpharmacologic lifestyle therapies have several important roles in both hypertensive and nonhypertensive individuals. In hypertensives, they can serve as initial therapy in Stage 1 hypertension before the addition of medication and as an adjunct to medication in persons already on drug therapy. Also in hypertensives with controlled blood pressure, lifestyle therapies could facilitate either decreased medication or even withdrawal. In nonhypertensives, lifestyle therapies have the potential to prevent the onset of hypertension, and more broadly to reduce blood pressure and thereby lover the risk of atherosclerotic cardiovascular disease in the general population (Appel, 1999). The new category, "prehypertension", introduced in the 2003 JNC 7 report, a systolic blood pressure between 120 and 139 or a diastolic between 80 and 89 , signifies this relationship. Given that the risk for of cardiovascular damage occurs at the 115 $\mathrm{mmHg}$ systolic level and most individuals do not see a healthcare provider until damage occurs, education must occur much sooner then later. Pre-hypertension is not considered a disease category but is useful in identifying individuals at risk for developing hypertension and provides an advanced opportunity to intervene with lifestyle modifications possibly before damage occurs and the high costs of treatment abound.

Primary prevention of hypertension must include effective public health interventions that will effect the population as a whole. Successful 
interventions to decrease the public health burden must include: (1) the will to act by clinicians, medical practitioners, and healthcare administrators and purchasers; comprehensive lifestyle programs, including lifestyle and clinical interventions, especially concerning the population 50 and older; (3) building and sustaining broad-based initiatives and commitment to these objectives by public and private sector partners at the national, State, and local levels; (4) prevention and reduction of excess body weight; (5) further promotion of the Dietary Approaches to Stop Hypertension (DASH) eating plan, including increased intake of fruits and vegetables, low-fat dairy products and whole grains; (6) strengthening the link between nutrition and physical activity in health promotion; (7) moderation of alcohol intake; and (8) decreasing the population's intake of sodium, especially exposure in the food supply. This essay will focus primarily on dietary sodium as one important lifestyle intervention.

The potential for having a major impact on the health of older individuals has changed dramatically in recent years, and the ability to translate the success of various intervention programs from observational and clinical trials into the community is a challenge. The extensive epidemiology and clinical research in the past 30 years has provided a strong evidence-based framework for application of preventive services for older individuals. The application of the knowledge obtained from these studies can have a very substantial impact on reducing morbidity and disability among older individuals.

Over the past two years, the Center for Healthy Aging (CHA), University of Pittsburgh, has been testing the use of preventive services and impact of lifestyle changes by promoting the " 10 Keys to Healthy Aging" in an at-risk community of older adults in Allegheny County. The CHA was established within the Graduate School of Public Health in September 2001 and is a Prevention Resource Center (PRC) supported with funds from the Centers for Disease Control (CDC). The CHA is highly collaborative within both the public health community and the community-at-large in order to compliment the already established flow of patient care and community programs in aging. The CHA has been focusing its efforts primarily in the McKeesport area of Allegheny County, Pennsylvania (PA). This city has a population of approximately 25,000 and represents a high risk population adversely affected by the closing of the steel mills and subsequent high unemployment, low education, low socioeconomic status (SES), and an aging population with substantial out-migration of younger individuals. Overall, the population of Western Pennsylvania, Allegheny County, has the highest percentage of older individuals in the United States, recently surpassing Dade County, Florida in terms of the percentage of the population over the age of 65 .

The most important determinants of adverse health outcomes among older individuals are the levels of specific risk factors and adherence to therapy. A major determinant of risk factors among these older individuals is the level of education. Education is more strikingly related to health than to either race or ethnicity, especially among the oldest of the old (Manton and Stallard, 1997). Thus, a major focus of the health program in Western Pennsylvania has been to reach the less educated, lower income, high-risk populations.

The CHA has developed an active Core Demonstration Project to test different health intervention models that could be adopted by community organizations. The target population includes men and women aged 65+ with no significant disability or difficulty with mobility. This Demonstration Project is a randomized trial comparing a more intensive healthy lifestyle intervention model with emphasis on control of hypertension and diabetes; to a brief health education model with evaluation and referral to healthcare providers and community partners. Both groups have access to a health counselor who assesses each individual based on the 10 keys to healthy aging, counsels based on determined goals, and performs follow-up assessments. Specifically, the Core Demonstration Project examines the impact of these two approaches on the following: reduction in risk factors for CVD, increase in screening and treatment of specific cancers, 
improvement in the prevention and treatment of osteoporosis and sarcopenia, increase in the rates of influenza and pneumonia vaccination, increase in levels of physical activity, decrease in depression, and improved nutrition.

The goals of the Core Demonstration Project are fourfold: (1) to train and use Health Counselors to help compare an intensive intervention program in nutrition, exercise, social support, depression and cognition to a brief less costly model of evaluation and referral; (2) to utilize the above as a training resource for CHA staff, community partners, University of Pittsburgh partners, and graduate students; (3) to train Community Ambassadors in the applications of the interventions including screening, behavioral approaches and evaluation; to further use the Community Ambassador program to reach more community participants, especially those who are very hard to recruit, such as low income, low education and minorities; and (4) to use the Core Demonstration Project as a viable training model for healthy aging for other PRCs, health departments and aging programs, both in the community and across the country.

There will be both intermediate and long-term outcomes assessed in both treatment groups. Overall, it is expected to see better adherence to therapy for diabetes and hypertension, an increase in the rate of screening for cancer and an increased use of flu and pneumonia vaccination from baseline (Table 2). In the intensive healthy lifestyle group it is expected to see an additional improvement in blood pressure, diabetes control, and other risk factors (lipids), as well as improvement in physical, cognitive and social functioning.

Table 2

Expected Outcomes Healthy Lifestyle as Compared to Brief Health Education

\begin{tabular}{|c|c|}
\hline Keys/Intervention & Outcomes \\
\hline $\begin{array}{l}\text { 1. Blood pressure, } \\
\text { diabetes and lipid } \\
\text { control }\end{array}$ & $\begin{array}{l}\text { Decreased incidence of stroke and vascular dementia. } \\
\text { Decreased incidence of myocardial infarction, congestive heart failure and renal } \\
\text { failure. } \\
\text { Decreased rate of hospitalization for myocardial infarction, congestive heart } \\
\text { failure and renal failure. }\end{array}$ \\
\hline Cancer screening & Decreased morbidity and mortality from colon, prostate and breast cancer. \\
\hline Immunization & Decrease hospitalization for pneumonia. \\
\hline $\begin{array}{l}\text { Physical activity and } \\
\text { functioning }\end{array}$ & Decrease in incident disability, nursing home placement and hospital days. \\
\hline $\begin{array}{l}\text { 5. Cognitive/social } \\
\text { functioning }\end{array}$ & $\begin{array}{l}\text { Decrease in disability, nursing home placement and hospital days. } \\
\text { Increased treatment for depression, decreased hospitalization for depression and } \\
\text { decrease in suicide. }\end{array}$ \\
\hline \multirow[t]{3}{*}{ 6. $\quad$ Nutrition } & Decrease in urine sodium excretion in hypertensives. \\
\hline & $\begin{array}{l}\text { Decrease in saturated fat intake and increase in fruits and vegetables (food } \\
\text { frequency). }\end{array}$ \\
\hline & $\begin{array}{l}\text { Decreased weight by } 5 \% \text { if BMI over } 30 . \\
\text { Further improvement in BP and Diabetes control. }\end{array}$ \\
\hline Activity & Increased walking frequency and duration. \\
\hline \multirow[t]{2}{*}{ 8. Physical function } & Increased endurance (corridor walk). \\
\hline & $\begin{array}{l}\text { Increase lower extremity function (lower extremity battery). } \\
\text { Increased lower extremity strength. }\end{array}$ \\
\hline Cognitive function & $\begin{array}{l}\text { Increased cognitive scores (MMSE, DSST), or slower decline in scores over } \\
\text { time. }\end{array}$ \\
\hline $\begin{array}{l}\text { 10. Social function and } \\
\text { depression }\end{array}$ & $\begin{array}{l}\text { Decreased CES-D score and other. } \\
\text { Increase social network size. }\end{array}$ \\
\hline
\end{tabular}


The Core Demonstration Project could have a major impact on the health of the growing population of older individuals in the United States. The methodology first involves both community partners and potential participants in the program as Ambassadors to recruit and deliver the preventive approaches. It does not require costly medical treatments. All approaches except for behavioral interventions are covered by Medicare and related health insurance and many of the behavioral interventions are likely to be covered in the future if demonstrated to be efficacious.

The Health Counselor training is modeled on the many years of success of health counselors in both infection and chronic disease, especially by health departments and the CDC. The intervention is proven to be efficacious and safe. The intensity of the intervention is consistent with the goals of the prevention program in the elderly. If the Core Demonstration Project is successful, it can easily be exported to other PRCs, health departments across the country through relatively simple and low-cost training programs. The CHA works within the community to promote health and to increase the understanding of the value of preventive services compared to invasive, expensive, and residential clinical services that are used after a person gets sick. The community outreach program plan has the following objectives:

- To improve community public health practice and enhance local, regional, and state public health efforts.

- To establish the concept of Healthy Aging as a theme and as a public health goal for the community.

- To disseminate quality prevention education materials to the community.

- To establish collaborative and meaningful program activities within the community of patients, providers, researchers, educators and policy makers.

\section{Review of Literature}

Looking back on the evolution of high blood pressure prevention and treatment, the foremost clinical trials focused on the benefits of antihypertensive drug therapy in patients 60 years and older with established hypertension. One study in particular, the 1991 Systolic Hypertension in the Elderly Program (SHEP) (SHEP Cooperative Research Group, 1991), assessed two antihypertensive medications in the reduction of the risk of nonfatal and fatal stroke in isolated systolic hypertension. In this study, the use of antihypertensive stepped-care drug treatment significantly reduced the risk of total stroke and coronary death.

Despite the repeated demonstration of the reduction, not elimination, of risk for stroke and coronary heart disease with antihypertensive medications, growing evidence supported further investigation of non-pharmacologic approaches to prevent, treat and manage hypertension. Such interventions were thought to possibly delay or avoid the need for drug therapy. Early studies demonstrated a relationship between sodium, potassium and blood pressure, with blood pressure increasing as sodium increased, although with more potassium blood pressures were lower. This relationship has also been demonstrated at the international level with the INTERMAP study (Zhou et al., 2003), comparing nutrient intakes among China, Japan, United Kingdom, and the U.S. The role of sodium and potassium are key in maintaining cellular homeostasis. The efficacy of utilizing nonpharmacologic interventions to lower blood pressure began over two decades ago with studies of benefits of specific nutrients, including calcium, magnesium, potassium and sodium. In Phase I of the Trials of Hypertension Prevention (TOHP) (Whelton et al., 1997) completed in 1992, the blood pressure-lowering effects of seven nonpharmacologic interventions (weight loss, sodium reduction, stress management, and supplementation of calcium, potassium, magnesium, and fish oils) were examined in middle-aged adults without prior hypertension. Results at six and 18-months demonstrated that sodium reduction and weight loss were well-tolerated and produced significant declines in systolic and diastolic blood pressures, while none of the other interventions were significant. In Phase II of the trial (Hebert, Bolt, Borhani, Cook et al., 1995), sodium and weight loss were examined more 
closely to test if weight loss alone, sodium reduction alone, or the combination of the two were beneficial in reducing blood pressures in moderately overweight, normotensive individuals. Results showed sodium and weight loss, both individually and in combination, were effective in lowering systolic and diastolic blood pressure. Compared to the usual care group, blood pressure decreased 3.7/2.7 $\mathrm{mmHg}$ (systolic/diastolic) in the weight loss group, 2.9/1.6 $\mathrm{mmHg}$ in the sodium reduction group, and $4.0 / 2.8 \mathrm{mmHg}$ in the combined group at six months. Short-term results (6-months) were more significant but incidence of hypertension remained reduced among longer follow-up periods (Herbert et al, 1995; Lasser, Raczynski, Stevens, Mattfeldt et al., 1995; TOHP Collaborative Research Group, 1997).

As the efficacy of sodium reduction and weight loss were proven, further studies began to examine the effects of lifestylenonpharmacologic interventions in combination with antihypertensive drug therapy. New recommendations from the $\mathrm{JNC} \mathrm{V}$ (JNC 5 Committee, 1988) emphasized the importance of lifestyle factors such as weight loss, sodium reduction, decreased alcohol intake and increased physical activity. Little was known about whether the effect of the multiple interventions was additive in blood pressure lowering or whether patients would be able to make several lifestyle changes simultaneously and then maintain them for long periods of time. The Treatment of Mild Hypertension Study (TOMHS) (Elmer, Grimm Jr., Laing, Grandits et al., 1995) was a randomized intervention in 1992 consisting of five different antihypertensive medications (or placebo) and a lifestyle intervention program to reduce weight, sodium, and alcohol, and to increase physical activity. Results showed substantial blood pressure changes, both short and long-term, in all intervention groups and demonstrate the important role of lifestyle interventions as the initial treatment for borderline and stage 1 hypertension and as an accompaniment to treatment with antihypertensive medications for the control of hypertension.
Both TOHP and TOHMS interventions focused on blood pressure lowering in mild and nonhypertensives, in middle-aged adults. The Trial of Nonpharmacologic Interventions in the Elderly (TONE) (Whelton, Appel, Espeland, Applegate et al., 1998), completed in 1996, was the first trial of sufficient size, duration and power to provide convincing evidence regarding the feasibility, efficacy, and safety of dietary lifestyle interventions, including reduced sodium intake combined with weight loss, as a means to control high blood pressure and decrease the need for antihypertensive medication in older persons (60-80 years) with hypertension. In the group that lost weight and reduced their salt intake, about half were able to stop and remain off medication versus about one third of those who received single interventions.

A large body of evidence from intervention and observational studies has accumulated to show that lifestyle and dietary factors influence blood pressure, and that altering specific nutrients can lower blood pressure in people with and without hypertension. When the updated JNC VI (JNC 6 Committee, 1997) was published, the committee extended its recommendations to include increased potassium intake for control of high blood pressure. In addition, the guidelines also recommended a healthy dietary pattern called the Dietary Approaches to Stop Hypertension (DASH) that is rich in fruits, vegetables, whole grains and low-fat dairy products, and reduced in saturated fat, total fat and cholesterol.

DASH continues to be a prominent force in the prevention and treatment of hypertension and lowers blood pressure overall in all individuals, men and women of all ages, and individuals on blood pressure medication as well as individuals with optimal blood pressure. In this eight-week feeding study, a "combination" diet that was high in fruits, vegetables, low-fat dairy products, whole grains, poultry, fish, and nuts and low in fats, red meat, and sweets produced relatively large decreases in blood pressure. The DASH trial (Appel et al., 1997) showed that in addition to calorie balance and intake of sodium chloride and alcohol, multiple nutrients influence blood pressure. The blood pressure reduction averaged $11 / 6 \mathrm{mmHg}$ among those with hypertension and 
4/2 $\mathrm{mmHg}$ among those without hypertension, with the greatest reduction in black hypertensive persons. DASH demonstrates that the blood pressure results were attributable to the influence of multiple nutrients. Compared with the control diet, the DASH diet was higher in calcium, lower than average in sodium (3000 $\mathrm{mg} / \mathrm{d}), 173 \%$ higher magnesium, $150 \%$ higher potassium, $240 \%$ higher fiber, and $30 \%$ higher protein. This eating plan has been called a diet for all diseases because it has proven to also decrease the risk for cancer, diabetes, heart disease, osteoporosis, macular degeneration, inflammation and age-related memory loss (secondary to a reduction in blood pressure).

An ancillary study published in 2001, the DASH-Sodium trial (Sacks, Svetkey, Vollmer et al., 2001) provided a special opportunity to demonstrate the blood pressure responses to changes in salt intake in a large study sample consuming a controlled diet. This 14-week, multi-center outpatient feeding study examined effects of sodium intake on blood pressure in participants given either a control diet, similar to what many Americans consume, or the DASH diet, each delivered at three different levels of sodium, fed in random order. The trial, which compared group mean changes, demonstrated that the blood pressure lowering effect of the DASH eating pattern was more effective when provided in conjunction with a low-sodium diet in people with hypertension.

Given the success of the DASH studies, the PREMIER (Svetkey, Harsha, Vollmer, Stevens et al., 2003) randomized trial was designed to further test a multi-component lifestyle intervention (DASH combined with weight loss, sodium reduction, increased physical activity, and limited alcohol intake) on blood pressure in demographic and clinical subgroups, in individuals aged 25 and older. Participants with above-optimal blood pressure through stage 1 hypertension were randomized to an Advice Only group or one of two behavioral interventions that implement established recommendations (Est) or established recommendations plus DASH diet (Est plus DASH). The PREMIER trial documented that individuals with above-optimal blood pressure, including stage 1 hypertension, can make multiple lifestyle changes that lower blood pressure and control hypertension (Writing Group of the PREMIER Collaborative Research Group, 2003). Both of the PREMIER behavioral interventions accomplished substantial weight loss, reduced sodium intake, and increased physical fitness. At six months, weight reduction was $4.9 \mathrm{~kg}$ and $5.8 \mathrm{~kg}$, urinary sodium decreased by $32 \mathrm{mmol} /$ day and $33 \mathrm{mmol} /$ day, and heart rate decreased by 8 beats/minute and 9 beats/minute, in the established and established plus DASH respectively. Participant goals for both the intervention groups were as follows: (1) weight loss of at least 15 pounds $(6.8 \mathrm{~kg})$ at six months for those with a BMI of at least 25, (2) at least 180 minutes per week of moderate intensity physical activity, (3) daily intake of no more than $100 \mathrm{mmol}(2300 \mathrm{mg})$ of dietary sodium, and (4) daily intake of 1 ounce or less of alcohol (two drinks) for men and $1 / 2$ ounce of alcohol (one drink) for women. Neither hypertension status nor age was a significant determinant of lifestyle change. Similarly, the PREMIER data suggest that both older and younger individuals succeeded with multiple, simultaneous lifestyle changes (Svetkey, Erlinger, Vollmer, Feldstein et al., 2005). Notably, in all groups the reduction in sodium intake was not sufficient to achieve the PREMIER (and JNC-VII) goal of no more than 100 mmolday. This goal, however, was reached in less than $50 \%$ of individuals in each racesex group, regardless of the treatment group even with ongoing counseling and staff support.

On average, most Americans consume substantially more sodium than physiologically needed, only about $500 \mathrm{mg}$ per day to replace necessary losses (Denton, 1982). Sodium is the principal cation of the extracellular fluid and functions as the osmotic determinant in regulating extracellular fluid volume and thus plasma volume. Excess sodium thus causes blood volume to increase in blood vessels, putting pressure on blood vessel walls and increasing blood pressure. With increasing age, the body is more likely to retain water, concurrently increasing the risk for high blood pressure. 
Recently released, the 2005 Dietary Guidelines (U. S. Department of Agriculture, 2005) for Americans summarize and synthesize the latest scientific evidence for lowering the risk of chronic disease and promoting health through diet and physical activity. First published in 1980, the guidelines are updated and published every five years and target the general American public over two years of age. The process of developing the guidelines is a joint effort of the U.S. Department of Health and Human Services (HHS) and the USDA. The information in the guidelines is useful for the development of educational materials and aids policymakers in designing and implementing nutrition-related programs. New items in 2005 include measuring in cups, rather than "servings"; discretionary calorie allowance in the USDA Food Guide and DASH eating plan; recommendations for special population groups; and calorie requirements based on gender, age, and level of physical activity.

The prior 2000 guidelines for sodium intake broadly recommended to choose and prepare foods with less salt. The 2005 guidelines specifically recommend 2,300 milligrams $(\mathrm{mg})$ or less per day for most adults. This is equivalent to only one teaspoon of salt. Many will benefit from reducing intake even more. The guidelines suggest $\leq 1,500 \mathrm{mg}$ per day for individuals at increased risk, individuals with hypertension, African Americans, and older adults. The Institute of Medicine also supports this recommendation but stresses that eating more than $1,500 \mathrm{mg}$ is not recommended for anyone (Panel on Dietary Reference Intakes for Electrolytes and Water, Standing Committee on the Scientific Evaluation of Dietary Reference
Intakes, Food and Nutrition Board, 2004). It also states that people over 50 should strive to eat just $1,300 \mathrm{mg}$ per day, and those over 70 just 1,200 mg. The Institute of Medicine provides unbiased, evidence-based, and authoritative information and advice concerning health and science policy to policy-makers, professionals, leaders in every sector of society, and the public at large. One well known publication of the institute is the Dietary Reference (DRI) reports that suggest upper and lower references levels of nutrients. According to the NHANES 1999-2000 survey data, Americans over the age of 60 are currently consuming, on average, $2,940 \mathrm{mg}$ of sodium a day, 2,532 $\mathrm{mg}$ (females) and 3,447 mg (males) (Figure 2). Thus, the average older adult is consuming more than double the sodium than the recommended level and these estimates have increased slightly from the NHANES 1994-1996 survey data (Wright et al., 2003). The estimates of dietary data from the NHANES survey consisted of a single 24-hour dietary recall and do not include discretionary sodium, such as salt added at the table. It is important to note that the assessment of sodium intake is especially complex because of the variety and nature of dietary sodium sources in the U. S. Sodium occurs naturally in some foods, such as milk and fresh meats, and is added to foods during processing, preparation and at the table. Although not usually taken into account in calculating total intake, sodium is also found in water, water-softening agents, dietary supplements and over the counter medications, such as antacids (Fregley, 1983; Mattes and Donnelly, 1991). Underreporting of intake, especially sodium, is another very common limitation of food questionnaires (Black and Cole, 2001). 


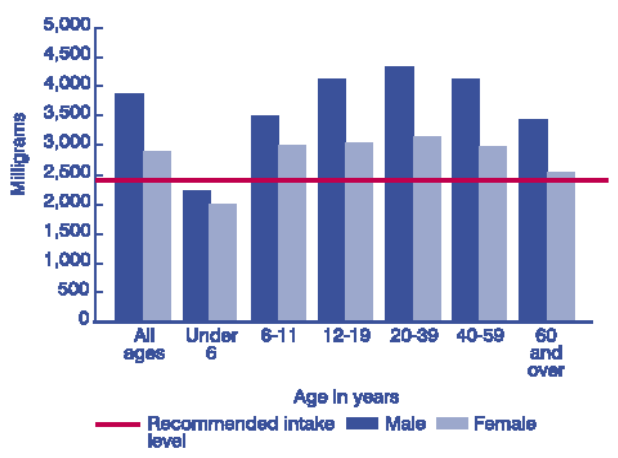

Figure 2

Dietary intake of sodium for U.S. population, NHANES 1999-2000

National databases are continually released to report food sources of nutrients of a large group of U.S. adults, aged 19 and older. The USDA Continuing Survey of Food Intake (CFSII) measures and identifies major food sources of 30 nutrients actually eaten by individuals. The survey records food intake over a specific period of time (one day in 1994-96 using a single 24hour dietary recall). The survey also collects demographic information, such as household size, income, race, age, and sex, and information on where a food was purchased, how it was prepared, and where it was eaten, in addition to food-intake data. The CSFII provides information for use in policy formation, regulation, program planning and evaluation, education, and research. Food sources of sodium are described in Table 3. The top ten sources have minimally changed from 1989-1991 (USDA Agricultural Research Service, 1996) to 1994-1996 (USDA Agricultural Research Service, 2000). Minor shifts were tomatoes, cakes/quick-breads, and cold cuts. Excluding milk, nearly all of the top sources are processed foods.

Table 3

Food sources of sodium among U.S. adults (from 1994-1996 and 1989-1991

Continuing Survey of Food Intake)

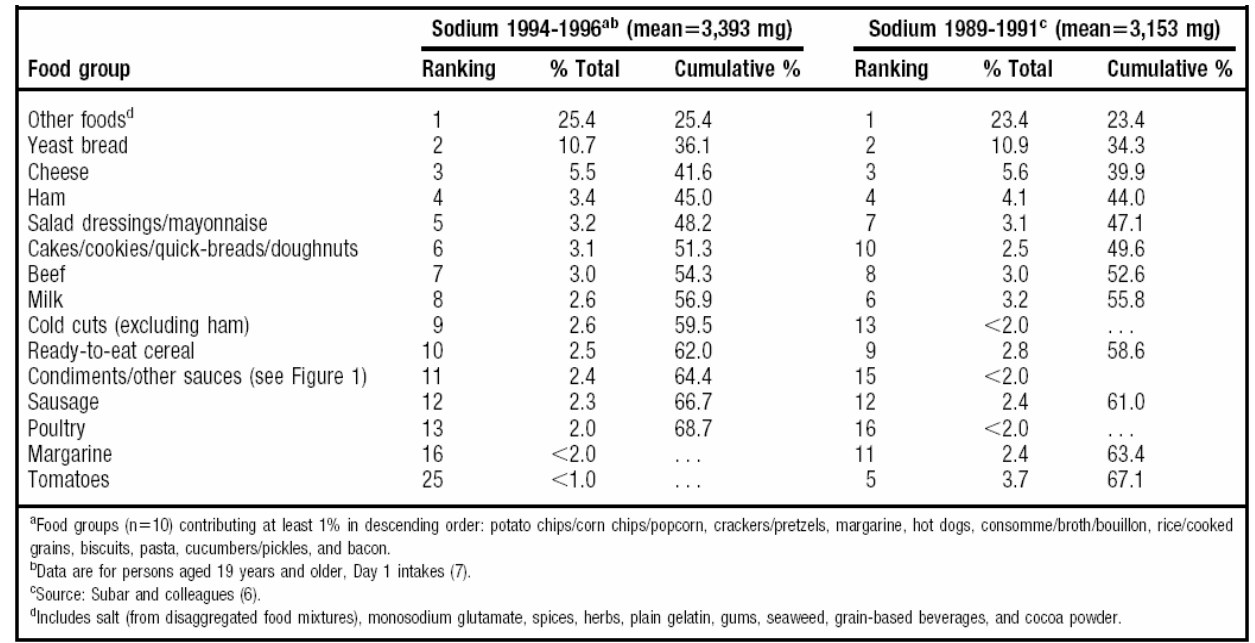


Few research studies have examined food group sources of sodium in older adults. One study assessed current dietary patterns of older adults with hypertension selected from a sample of rural elderly persons, compared them with the patterns of older adults without hypertension, and described their specific hypertension-related food group patterns (Lancaster et al., 2004).

Food and nutrient intake was measured using five 24-hour recalls conducted by telephone over a year. Nutrient data were averaged across five days to obtain energy, fat, calcium, magnesium, potassium, and sodium intakes. In regards to sodium intake, results showed that the top five food group sources of sodium for hypertensives were (1) processed meats, (2) pancakes, waffles, and french toast, (3) vegetable sauces and juices, (4) breads, and (5) cheese. The mean dietary sodium intake was $2,962 \mathrm{mg}$ and $2,540 \mathrm{mg}$ for non-hypertensives and hypertensives respectively. The nutrient and food group intakes of the two groups were similar, except that those with hypertension ate less sodium and fewer grain and meat servings. One limitation of this study is the absence of specific food group sources of the nutrients in the non-hypertensive individuals. Comparisons between the two groups could help focus the dietary intervention efforts of older adults, with and without hypertension, at the community level.

Accurately assessing dietary intake in populations has numerous methodological limitations. Although several validation and reproducibility studies have been performed on any given food questionnaire or dietary intake tool, population demographics and personal error pose indeterminate factors. Seasonal affects, personal memory of past intake, underreporting, unwillingness to keep daily food records, and continual modifications in the food industry represent just a few factors affecting accuracy of intake. Assessing dietary intake is generally utilized in research protocols to better understand the role of diet in health and disease. Selecting a tool with proven validity and reliability is critical for scientific research. The Block Food Frequency Questionnaire (BFQ), developed at the National Cancer Institute at UC Berkeley, is in use by over 700 registered research and public health groups, in university and public health settings around the country. Continually updated and improved, this particular tool provides not only specific nutrient intake but also 83 possible food group sources of these nutrients. This tool was used in CHA to collect dietary data.

Sodium is relatively well reflected in biochemical measures, because the main route of sodium disposal is through urine, with only small losses through skin via perspiration either from physical activity or warm climates (Gibson, 1990; Sanchez-Castillo, Branch, and James, 1987). For this reason, urinary sodium is collected from subjects to provide a more reliable estimate of sodium intake. Because of high day-to-day within-person variability in intake, it is preferable to include several 24-hour urine collections to accurately estimate an individual's usual sodium intake. Estimates range from as few as five days (He et al., 1993) to as many as 14 days (Liu, K. \& Stamler, J., 1984), when the desired accuracy is within $10 \%$ of true intake. A single 24-hour urine collection can be used to provide a rough estimate of daily sodium excretion and therefore daily sodium intake. A single 24-hour urine adequately reflects the previous day's dietary sodium intake and can be a good estimate for groups (Bingham, Williams, Cole, Price, Cummings, 1988). Accuracy in 24-hour urine measurements may occur because study participants may alter their usual food and beverage intake on collection dates and may not collect all urine from a 24-hour period. One important strength of measuring urinary sodium to assess intake over self-reported questionnaires is that it reflects sodium from all sources. Weak relationships between dietary and urinary sodium is attributed to the poor assessment of salt intake by dietary assessment methods, the lack of inclusion of foods prepared with salt in food-composition tables, and the high withinperson variability of urinary sodium (Caggiula, Wing, Nowalk, Milas, Lee, Langford, 1985; Schachter, Harper, Raden, Caggiula, McDonald and Diven, 1980). Although difficult to collect, this "snap-shot" of sodium intake may be useful as a screening device to identify individuals atrisk for hypertension based on their dietary 
sodium levels. Urinary sodium does not, however, help determine dietary sources of sodium. Efforts to improve collection procedures for both diet and urine are critical in order to improve accuracy and together are useful to target individuals and food sources.

The goal of this essay will be to describe the sodium intake of a group of older adults by comparing self-report to urinary measures, determine the major food group sources of sodium, and demonstrate the need for a stronger public health message to link less dietary sodium to improved blood pressure control.

\section{Methodolgy}

The CHA used the master list of all Allegheny County residents to identify random households of potentially eligible participants based on address, age, and race. A letter describing the study was mailed to the resident's home, along with a returnable card for those who prefer not to be contacted. Approximately two weeks later after the mailing, residents were contacted by telephone recruiters. These individuals provided a description of the program, explained the benefits, determined eligibility, and scheduled individuals to the CHA center to attend an initial appointment where multiple baseline measurements were collected. The criteria for eligibility and randomization for the CHA demonstration project are as follows:

- $65+$, Medicare Part A and B or other type of insurance;

- Able to function outside the home, defined as no difficulty with community ambulation, defined as no difficulty walking $1 / 4$ mile or climbing 10 step by self-report. No need for cane or walker;

- Not under active treatment for cancer (defined as surgery, radiation or chemotherapy in the past year. (Exceptions: basal or squamous skin cancer and maintenance treatments);

- No other advanced condition such as lung disease requiring oxygen, congestive heart failure requiring oxygen or IV medication, renal failure requiring dialysis, stroke with disability or other advanced condition;
- No plans to move in the next 2 years;

- Cognitively intact, able to understand the purpose and goals of the project and the tasks involved in participating, and willing and able to give informed consent;

- Interested in participating in the program;

- Able to identify a physician or be willing to have the study one selected; and

- Physician permission to join.

After eligibility was re-confirmed, other data was collected including; (1) a complete medical history of chronic illness and cancer; (2) screening tests for breast, prostate, and colon cancer, bone density, and immunizations; (3) and a basic health status questionnaire and risk factor analysis. Blood pressure was measured and medications were identified. Other baseline blood tests included a complete lipid profile, hemoglobin A1-C, fasting blood glucose, and serum creatinine. Height, weight, waist circumference, and weight change history were also obtained. Cognitive function tests and measurements of depression were measured by a modified mini mental, MAQ and CES-D. Muscle strength, balance and gait speed were measured by a Lower Extremity Battery Test.

At the end of two baseline clinic visits, individuals were randomized to either intensive healthy lifestyle or brief health education group. The randomization was conducted through the Epidemiology Data Center in the Department of Epidemiology at the Graduate School of Public Health. The healthy lifestyle group received the same health counselor support and counseling as those randomized to the brief health education group, with the addition of behavioral interventions in exercise, nutrition, and in social/cognitive enhancements. A brief Block FFQ (BFQ) was obtained at baseline for all randomized participants to assess dietary intake. Hypertensives (SBP $>140 \mathrm{mmHg}$ or on blood pressure medications) were identified at randomization and were asked to also collect one 24-hour urine at baseline. Only the hypertensives randomized to the healthy lifestyle group were asked to collect a 24-hour urine and BFQ at each follow-up visit (six-month, 12month and 18-month). An intention to treat 
model was used to analyze all data whether participants were randomized to the intensive intervention program or not. One goal of this paper is to compare the sodium intake of all participants who completed a BFQ to the subsample of hypertensives $(n=214)$ to determine the best estimate of dietary and urinary sodium intake.

To improve the completeness of the urinary data, participants were telephoned and reminded three days prior to their visit to collect the 24hour urine. When the participant arrived with their bottle(s), instructions were reviewed to determine whether the sample was complete and their ID number was placed on the 24-hour urine list to identify the start and stop date of the collection. If the urine collection bottle was not returned at the time of the visit, the importance of the collections was reviewed and participants were asked to keep a 24-hour urine and return to the clinic within three days.

The BFQ was a self-administered tool to collect nutrition information over the past three months and the foods were categorized into 83 groups or items. The importance and rationale of the BFQ was explained to every participant. To promote accuracy and ease of the BFQ, the participants were given specific directions to think about what they ate, not what their family ate and not what they think they should have eaten, and to take their time to fill out the questionnaire themselves. Food illustration guides were provided to show examples of proper portion sizes.

The Key to Life Nutrition Program to lower systolic blood pressure was designed and delivered to hypertensive participants randomized to the healthy lifestyle group. Efforts to promote attendance to the program were encouraged. Overall, the nutrition program to lower systolic blood pressure used the core the findings from the Dietary Approaches to Lower Systolic Blood Pressure (DASH) that builds a lower-sodium eating pattern that advocates a diet rich in fruits, vegetables and low-fat dairy products. Weight loss was recommended in participants with a BMI over 30 and moderation in alcohol intake is advised to all. A professional nutritionist facilitated this program and included individual and group sessions. The intervention was initiated at randomization for each participant to promptly engage him/her in efforts to identify dietary sources of sodium at entry. After the individual session, participants were combined in a group that met weekly for ten weeks to learn about high blood pressure, strategies to lower blood pressure and establish personal goals. After the intensive ten weeks, the group met monthly for further motivation and adherence.

The intervention program incorporated the following components which are firmly grounded in behavior theory, namely, behavior modification, social learning theories, knowledge acquisition, skill development, selfmonitoring, stimulus control, stress management, social support, relapse prevention, social modeling, goal setting, and other selfmanagement strategies. Specifically, participants were taught how to modify their physical and social environment and their beliefs relating to eating. Since some individuals were on blood pressure medications already, the nutrition program also strived to improve adherence to medications. The nutrition intervention program incorporated dietary modifications that could simultaneously lower LDL cholesterol and improve blood glucose management. The goal was to maximize blood pressure response via a comprehensive lifestyle program that if successful, could be transported to the community. The demonstration project would ultimately compare the number of study participants whose blood pressure was less than $140 \mathrm{mmHg}$ between participants randomized to the intensive healthy lifestyle versus those assigned to the brief health education model.

\section{Results}

By the end of December 2003, 541 participants had been randomized (273 to the brief education group and 268 to the healthy lifestyle group). By the end of December 2003, 541 participants had been randomized (273 to the brief education group and 268 to the healthy lifestyle group). Baseline socio-demographics are: Mean Age 74.5 Years (range 65.0-95.5); Gender - 39.9\% Male, $60.1 \%$ Female; Race 5.9\% Non-white, 
94.1\% White; Marital Status - 60.4\% Married, $28.3 \%$ Widowed, $5.2 \%$ Divorced, .9\% Separated, 5.2\% Single; Education - 6.7\% Less than High School, 40.3\% High School, 53.1\% Greater than High School. A report on the percent of participants who are meeting each of the "10 Keys to Healthy Aging" goals is described in Table 4. Important for this paper is the fact that only $68 \%$ of participants in the demonstration project were meeting the systolic blood pressure goal of less than $140 \mathrm{mmHg}$ at entry into the study (baseline).

Mean baseline dietary sodium as estimated by the BFQ for the entire cohort was 1,796 mg per day compared to $1,821 \mathrm{mg}$ per day in hypertensives. At six months, dietary sodium intake reported via the BFQ in hypertensives $(\mathrm{n}=84)$ decreased to $1,525 \mathrm{mg}$ per day. Sodium added at the table or in cooking was not included in the analysis. At baseline, urinary sodium was 1.8 times higher $(141 \mathrm{mmol} / 24 \mathrm{hrs})$ than selfreported intake for hypertensives and decreased slightly to $130 \mathrm{mmol} / 24 \mathrm{hrs}$ at six months (Table $5)$. The correlation between dietary sodium and urinary sodium was weak $(\mathrm{r}=0.16)$ at baseline and remained weak $(r=0.23)$ at six months. At baseline, 521 individuals $(96 \%)$ completed a BFQ and 214 (83\%) of hypertensives collected a 24-hour urine. Attrition rates at six months were $33 \%$ (84) and 35\% (90) of hypertensives who collected a follow-up BFQ and 24-hour urine, respectively.

Table 4

Characteristics of variables at baseline $(n=541) ; \%$ meeting goals

\begin{tabular}{|l|r|}
\hline \multicolumn{1}{|c|}{ "10 Keys to Healthy Aging” Goals } & Overall \% (n=430) \\
\hline Control Systolic Blood Pressure $<140 \mathrm{mmHg}$ & $68 \%$ \\
\hline Maintain LDL Cholesterol to $<100 \mathrm{mg} / \mathrm{dL}$ for CAD and Diabetes & $54 \%$ \\
\hline Regulate Fasting Blood Glucose $<110 \mathrm{mg} / \mathrm{dL}$ & $86 \%$ \\
\hline Combat Depression $(\mathrm{CES}-\mathrm{D})<16$ & $95 \%$ \\
\hline Increase Physical Activity $>2.5$ hours/wk & $64 \%$ \\
\hline Participate in Cancer Screenings & $55.5 \%$ \\
Colonoscopy & $85.5 \%$ \\
Mammogram & $70 \%$ \\
\hline Get Regular Immunizations & $92 \%$ \\
Pneumovax & $79 \%$ \\
\hline Influenza & $95.6 \%$ \\
\hline Prevent Bone Loss and Muscle Weakness & $99.3 \%$ \\
\hline No Smoking & \\
\hline Maintain Social Contact & \\
\hline
\end{tabular}

Table 5

Dietary and urinary sodium levels at baseline and six months

\begin{tabular}{|l|l|l|}
\hline & \multicolumn{1}{|c|}{ Baseline } & \multicolumn{1}{c|}{ Six Months } \\
\hline Dietary Sodium (mg)-entire cohort & $1,796(\mathrm{n}=521)$ & NA \\
\hline Dietary Sodium (mg)-hypertensives & $1,821(\mathrm{n}=215)$ & $1,525(\mathrm{n}=84)$ \\
\hline Urinary Sodium (mmol/24 hrs)-hypertensives & $141(3,240 \mathrm{mg})(\mathrm{n}=214)$ & $130(2,990 \mathrm{mg})(\mathrm{n}=90)$ \\
\hline
\end{tabular}


The food group sources of sodium are summarized below in Table 6. For the entire cohort, the major food sources of sodium were soups, breads, tomato sauce, salad dressings, and prepared cereals. The sources for hypertensives were very similar to the entire cohort at baseline and with some reduction at six months did not change more or less. The top sources, soup, breads, tomato sauce, salad dressing, cereals, and sausage, contain various sodium compounds added during food processing. Milk and cheese include naturally occurring sodium.

Table 6

Top 10 food group sources of sodium at baseline and six months

\begin{tabular}{ll|l|l|}
\hline \multicolumn{1}{c|}{$\begin{array}{c}\text { Baseline Entire Cohort } \\
(\mathbf{n}=\mathbf{5 2 1})\end{array}$} & \multicolumn{1}{c|}{$\begin{array}{c}\text { Baseline Hypertensives } \\
(\mathbf{n = 2 1 4})\end{array}$} & \multicolumn{1}{c|}{$\begin{array}{c}\text { Six months Hypertensives } \\
(\mathbf{n = 8 4 )}\end{array}$} \\
\hline 1. & Vegetable soup & Vegetable soup & Vegetable soup \\
\hline 2. & Dark breads, whole wheat, rye & Dark breads, whole wheat, rye & Dark breads, whole wheat, rye \\
\hline $\begin{array}{l}\text { 3. } \\
\text { meat }\end{array}$ & Spaghetti w/ tomato sauce and & Spaghetti w/ tomato sauce and meat & Spaghetti w/ tomato sauce and meat \\
\hline $\begin{array}{l}\text { White bread, French, Italian, } \\
\text { etc }\end{array}$ & White bread, French, Italian, etc & Cooked cereal or grits \\
\hline 5. Salad dressing & Salad dressing & White bread, French, Italian, etc \\
\hline 6. $\quad$ Cornflakes & Cornflakes & Nonfat milk \\
\hline 7. & Nonfat milk & Nonfat milk & Salad dressing \\
\hline 8. & Cooked cereal or grits & Cooked cereal or grits & Cheese and cheese spreads \\
\hline 9. & Cheese and cheese spreads & Breakfast sausage & Breakfast sausage \\
\hline 10. & Breakfast sausage & Doughnuts, pastry & Raisin Bran Cereal \\
\hline
\end{tabular}

\section{Discussion}

A major objective of this essay was to examine a specific community intervention program piloted for older adults to help better understand the benefits and barriers of implementing a blood pressure and sodium reduction program. Additionally, this paper may help define the dietary sodium pattern of a select population of older adults in the community under study.

The total sodium intake and food group patterns were very similar between hypertensive and non-hypertensives. It is important to note that both groups are consuming far more sodium than the recommended levels for this at-risk population. Based on the baseline urinary sodium results, the sodium intake was approximately 160\% higher and 200\% higher than the 2005 Dietary Guidelines and Institute of
Medicine's recommendations, respectively, and significantly more than the minimum of $500 \mathrm{mg}$ physiologically needed. These results are not surprising given that most of the U.S. population is not meeting the recommended goals for sodium as reported by the NHANES 1999-2000 and CFSII 1994-1996. The levels of sodium reduction reported in controlled blood pressure and diet trials have been both significant and variable and none have reported long-term intakes below $100 \mathrm{mmol} / 24$ hours $(2,300 \mathrm{mg})$, even when dietary intake and subjects' adherence is consistently monitored. Despite the pleas of health experts to cut back, salt consumption has steadily drifted upwards since the 1970's (Briefel et al., 1995). The reduction in urinary sodium excretion in CHA participants compared to other intervention studies is summarized in Table 7 . While it was expected 
that changes in sodium response would not reach levels attained in controlled clinical trials, the rich experience of past research protocols provided a foundation that could be maximized and disseminated to the community.

Table 7

Baseline and 6-month urinary sodium values in lifestyle modification for blood pressure and sodium reduction interventions

\begin{tabular}{|l|l|c|c|}
\hline \multicolumn{1}{|c|}{ Study } & \multicolumn{1}{|c|}{ Treatment group } & $\begin{array}{c}\text { Baseline (mmol/24 } \\
\mathbf{h r s})\end{array}$ & $\begin{array}{c}\mathbf{6 - m o n t h s} \\
(\boldsymbol{\Delta} \text { in } \mathbf{~ m m o l} / \mathbf{2 4 h} \mathbf{h r})\end{array}$ \\
\hline CHA & All hypertensives & 141 & -11.4 \\
\hline TOHP II & Weight loss & 180.9 & -18.2 \\
\hline & Sodium reduction & 186.1 & -78.0 \\
\hline & Combined & 179.3 & -64.3 \\
\hline TONE (All participants) & Usual care & 188.0 & -27.6 \\
\hline \multicolumn{1}{|c|}{ (Overweight) } & Sodium reduction & 150 & -46.6 \\
\hline TOMHS & No sodium reduction & 138 & -1.4 \\
\hline PREMIER (>50 years old) & Sodium reduction & 135 & -28.7 \\
\hline & All & 161 & -12.5 \\
\hline & Advice & 168.2 & -17.7 \\
\hline & Established & 164.9 & -34.1 \\
\hline & Est + DASH & 173.5 & -41.2 \\
\hline
\end{tabular}

The CHA is a community-based demonstration project that focused on the overall health of older adults through prevention and awareness across all of the "10 Keys to Healthy Aging". The goal to reach a systolic blood pressure of $\leq 140 \mathrm{mmHg}$ represented just one important key out of several keys that volunteers may have needed to modify to lower their risk of disease. All hypertensive individuals randomized to the intensive lifestyle group were invited to attend a comprehensive nutrition program to lower systolic blood pressure and attendance was voluntary. This was a significantly different research design than what was used in the DASH. DASH was a controlled feeding program where foods were supplied and the follow-up was relatively short. Adherence is always superior in this study design. Attrition, adherence and recovery are key to the success of clinical trials that boast major reductions in sodium and blood pressure. Implementing an effective community intervention, especially with the older adult population, has numerous barriers that must be overcome. First, many older adults have substantial co-morbidities, creating more complications, hospitalizations and absences to attendance at intervention programs. Second, many are also taking care of sick loved ones, leaving less time for personal health. Third, it has been documented that the perception is that a low sodium diet is more expensive. Consumer education is key to positively shift these widespread perceptions. Fourth, there is substantial problem related to transporting older individuals to community programs. Without transportation, many older individuals cannot reach preventive health services and programs. Although the CHA provided transportation to and from intervention classes, the day and/or time of the classes may have conflicted with other activities and obligations. The CHA offered a variety of days and times for intervention classes to accommodate most individuals. Fifth, cognitive changes and memory losses that can accompany aging may interfere with the ability of some participants to select lower sodium foods and/or modify recipes for sodium. Sixth is the 
misconception among some older individuals in the inevitability of aging and the belief that behavioral change in later life would be futile. The CHA places a strong focus on the benefits of prevention and the fact that the course of most chronic conditions can be improved with intervention at almost any age. This is a positive message that needs to be reinforced over and over again by health professionals so older adults elect to become more proactive in their health care.

Difficulties involved in the collection and measurement of total dietary and urinary sodium in this volunteer community sample do occur despite procedures to minimize errors. Only one 24-hour urine was obtained because the goal was to obtain a "best estimate" of sodium intake and to minimize burden. For some volunteers the sample collected may not represent a usual day. Multiple samples would have improved the accuracy, however obtaining multiple 24-hour urine samples in a free-living population is not an easy task and may discourage participants from volunteering. The intention to treat model data analysis included everyone randomized to the intervention, even those who never attended. Correlations between urinary and dietary sodium measurements were significantly weak at both baseline and six months. This poor relationship is not uncommon and occurs because of the imperfection in dietary intake tools in capturing all dietary sources.

The top food sources of sodium were also comparable to previous reports of adults. Given that many older adults are on a fixed, reduced income and must rely on shelf-stable and prepared foods that contribute significant sodium and for which alternatives are not readily available, this is not surprising. Sodium is: (1) commonly added to soups, tomato sauce, and salad dressings as a preservative; (2) used as a leavening agent, bacteria prevention, and flavor enhancer in breads and cereals; (3) acts as a agent for making meat proteins more soluble (sausage); (4) aids in fermentation, moisture control and emulsification in cheeses; (5) and naturally occurs in milk products (Crocco, 1982). Food group sources of nutrients, particularly sodium, are also difficult to measure, as how foods are grouped in the database varies by the measurement tool used. The BFQ used in CHA tracked 83 grouped food items and measured the previous three months of intake. Given the variability in commercial and restaurant versus home-prepared meals, seasonal food preferences, low and reduced sodium products, ability to recall intake for three months, and food processing overall, it is difficult to accurately capture the common food sources sodium in the U. S. diet. The BFQ was a reliable tool to report intake for groups of individuals. Tools like the BFQ can be used at the community level to help identify foods to target to reduce sodium intake. Results of this demonstration project suggest that further efforts to reduce sodium intake in this population of older adults are needed.

Adherence to lifestyle approaches that support blood pressure reduction is challenging. Combined with drug therapy, modest weight loss and sodium reduction has the potential to lower blood pressure levels in the population and prevent the significant economic and health burdens of hypertension. Even a small decrease of $2 \mathrm{mmHg}$ systolic measure at the population level could substantially decrease the incidence and magnitude of high blood pressure complications (Whelton et al., 2002). To reach the blood pressure goal of less than 140/90 $\mathrm{mmHg}$, the JNC VII, 2005 Dietary Guidelines, and Institute of Medicine all support the possible need for further reduction of sodium intake to $1,500 \mathrm{mg}$ for older adults and others at high risk. On the other hand, many health professionals believe the new sodium guidelines are an unrealistic goal for all consumers as these reduced levels have not been reached even in controlled clinical trials with maximum support and adherence counseling. Ambitious goals may be a step in the right direction, considering the epidemic of hypertension in the U.S. To achieve the Healthy People 2010 goal of increasing the proportion of persons $(20+)$ who consume less than 2,300 $\mathrm{mg}$ of sodium per day and less than $1,500 \mathrm{mg}$ for those at increased risk, the food industry must become a key target for change. An ambitious sodium goal could help Americans shift their sodium intake downward even if the goal is not reached. In older adults, efforts to 
lower blood pressure will require both nonpharmacologic and phamacologic approaches. The high costs and potential sideeffects of multiple medications, reliance on a fixed income, increased need for shelf-stable foods and decreased taste acuity create unique challenges in older adults. Many older adults decrease their food intake as they age and maintaining adequate energy is important with less sodium. The guidelines for most nutrients, including fat, saturated fat, and protein, are based on a specific calorie level. The question then becomes, should guidelines for sodium be universal or be adjusted to specific populations?

The high levels of hidden sodium in the manufacturing of processed and restaurant foods make it more difficult for consumers to eat less sodium. Some may be willing to read labels and select the lower sodium products. Restaurant foods do not list sodium and few restaurants have made efforts to reduce sodium. Public health messages to advise individuals on how to select and prepare foods with less sodium must be more widely available. The increase in foods eaten outside of the home, the desire for preprepared, quick meals, and the fact that the majority $(70 \%-80 \%)$ of the sodium in our diet is added to food during commercial processing and cooking (Figure 3) and convenience or processed foods, makes sodium reduction efforts a challenge (Mattes \& Donnelly, 1991; Whelton et al., 2002). Support from the food industry is key. A false sense of security seems to be evident in many individuals who underestimate how much sodium they are consuming because they think that just because they do not use added salt they are eating a diet low in sodium. Consumers need to understand the recommended guidelines and how to read a food label for the sodium content. In addition, many associate the sensory pleasure with salt with the taste they desire, and most individuals do not want to choose between health and taste. As the taste for salt is acquired, gradual, voluntary sodium changes in the supply can occur without people choosing to eat less sodium. Since preference for salt begins in childhood, efforts to eliminate salt in baby foods have been successful, however as these children become exposed to sodium in their environment their preference increases. Lower sodium alternatives have been added to menus and grocery store shelves yet consumer demand has not been successful to stimulate further production and profits. Positive incentives for food companies and restaurants to decrease the sodium content of their foods and develop less expensive, palatable low sodium products must be included in this strategy. Dictating legislation to assist with these goals may be resisted by companies where profit motives outweigh health concerns.

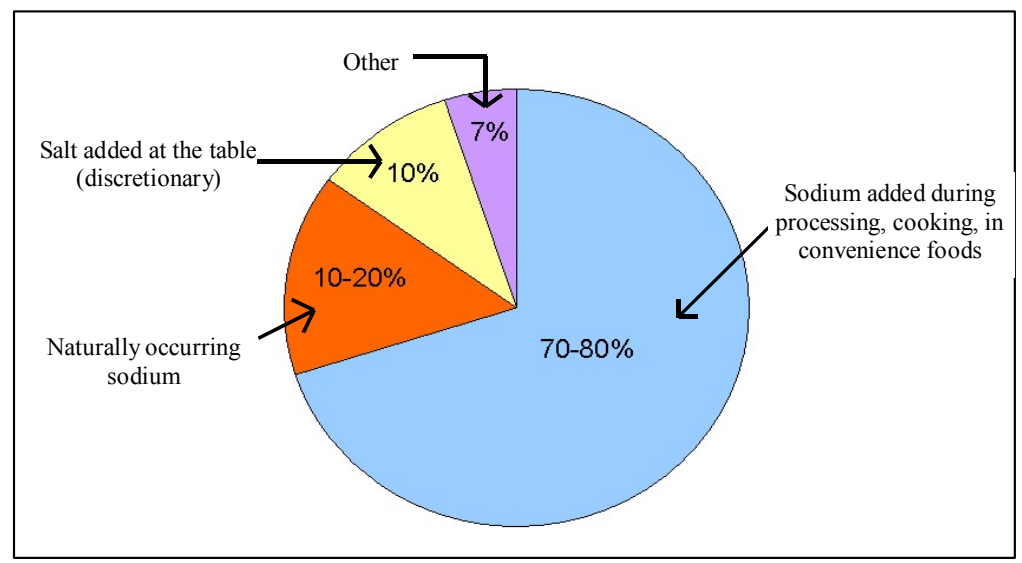

Figure 3

Dietary Sources of Sodium in the Typical U.S. Diet 
In November of 2002, the American Public Health Association adopted a policy calling for a $50 \%$ reduction in sodium in processed and restaurant foods over the next 10 years (American Public Health Association, 2002). If this policy is successful it could potentially save in the upwards of 150,000 lives per year from the complications of high blood pressure. This strategy has already been adopted in the United Kingdom by both the Department of Health and Food Standards Agency (He \& MacGregor, 2003). Several leading supermarkets and food manufacturers have begun to reduce the salt content of their products without advertising in the community. For example, a supermarket (Sainsbury's) in the UK has reduced sodium in its store brand products with the aim to reduce salt content by $10-15 \%$ without affecting taste or quality (J. Sainsbury plc, 2005). To date, it has already removed about 400 tons of salt per year across an array of products, without advertising and with minimal complaints from the consumers. Due to the increasing incidence of hypertension in the American and worldwide population, the demand for lower sodium products will hopefully soon become widespread. Given the substantial evidence of the benefits of a low-fat eating pattern and the recent enthusiasm of low carbohydrate diets, the concern for salt still ranks below fat and sugar. Notably, it took more than ten years for the industry to successfully market and sell low-fat products. Americans are more health-conscious today and have made significant changes in the intake of total fat and saturated fat (Wright, J. et al., 2003). On the other hand, the food industry continues to create "healthy eating" roadblocks by spending hundreds of millions of dollars a year exposing the public to conflicting messages which confuse even those who are health conscious. Hopefully the new blood pressure guidelines that emphasize optimal blood pressure levels, revised sodium recommendations and the increasing rate of hypertension may stimulate individuals to become more educated about the amount of sodium they are consuming and take action to eat less.

\section{Conclusion}

The purpose of this essay is to (1) describe the sodium intake of a group of older adults by comparing self-report to urinary measures; (2) determine major food group sources of sodium in a group of older adults; and (3) demonstrate the need for a stronger public health messages to link less dietary sodium to improved blood pressure control. Generally, the total sodium intake, based on the 24-hour urinary sodium, and the top food sources of sodium of the CHA cohort of older adults are similar with the intake of the U.S. population. Both hypertensives and non-hypertensives are consuming too much sodium and the top food sources supplying the majority of sodium are processed foods. Intervention efforts to reduce sodium must focus on less sodium added to processed foods. Support of the food industry is necessary.

Attainment of lower sodium levels to decrease blood pressure levels represents an important public health opportunity and challenge for the population as a whole, as most Americans are at increased risk for hypertension as they age. Due to co-morbidities in the older adult population, a more comprehensive intervention program must be employed to significantly impact blood pressure levels. Educational messages that inform the public about the major sodium food contributors to sodium and how to use food labels to identify and choose lower sodium foods is needed. Because the taste for salt is acquired over time, nutrition education must begin in the infancy and childhood stages. It may be very beneficial to intervene in younger generations in the pursuit to decrease the prevalence of hypertension in later life. Gradually decreasing the sodium added during food processing and in restaurants, as has been implemented in other countries, may be another safe public health intervention strategy to help meet the Healthy People 2010 intervention objectives that focus on reducing the incidence of hypertension and sodium intakes of the general population. An effective national effort aimed at the prevention of blood pressure must also involve strong and active support from healthcare providers. In turn, these improvements, along with the new blood pressure and sodium guidelines, will guide the population to attain the goal of a $20 \%$ 
reduction in heart disease and stroke-related mortality. Action that leads to the improved control and prevention of hypertension, and reduces the burden of heart disease and stroke, demands immediate and continued efforts that improve the economic implications, overall health and quality of life.

\section{References}

American Public Health Association. (2002). Reducing the sodium content in the American diet. Washington, DC: Author.

Appel, L. J. et al. (1997). The effect of dietary patterns on blood pressure: results from the dietary approaches to stop hypertension (DASH) trial. New England Journal of Medicine, 336, $1117-$ 1124.

Appel, L. J. (1999). Nonpharmacologic therapies that reduce blood pressure: a fresh perspective. Clinical Cardiology, 22(7), III-1-5.

Auchmutey, J. (2003). Stealth salt: Restaurants and food manufacturers need to shake the salt, doctors say. Atlanta Journal-Constitution.

Bingham, S. A., Williams, R., Cole, T. J., Price, C. P., Cummings, J. H. (1988). Reference values for analysis of 24-h urine collections known to be complete. Annals of Clinical Biochemistry, 25, 610-619.

Black, A. E., and Cole, T. J. (2001). Biased over- or under-reporting is characteristic of individuals whether over time or by different assessment methods. Journal of the American Dietetic Association, 101, 70-80.

Briefel, R. R. et al. (1995). Total energy intake of the population: the third national health and nutrition examination survey; 1988-1991. American Journal of Clinical Nutrition, 62, 1072S-1080S.

Caggiula, A. W., Wing, R. R., Nowalk, M. P., Milas, N. C., Lee, S., Langford, H. (1985). The measurement of sodium and potassium intake. American Journal of Clinical Nutrition, 42, 391398.

Crocco, S. (1982). The role of sodium in food processing. Journal of the American Dietetic Association, 80, 36-39.

Denton, D. (1982). The hunger for salt: an anthropological, physiological and medical analysis. Berlin: Springer-Verlag.

Elmer, P. J., Grimm Jr., R., Laing, B., Grandits, G. et al. (1995). Lifestyle intervention: Results of the treatment of mild hypertension study (TOMHS). Preventive Medicine, 24, 378-388.

Fregley, M. J. (1983). Estimates of sodium and potassium intake. Annals of Internal Medicine, 98, 792799.

Gibson, R. S. (1990). Principles for nutritional assessment. NY: Oxford University Press.

He, J. et al. (1993). Agreement between overnight and 24-hour urinary cation excretions in southern Chinese men. American Journal of Epidemiology, 137, 1212-1220.

Havas, S., Roccella, E., and Lenfant, C. (2004). Reducing the public health burden from elevated blood pressure levels in the United States by lowering intake of dietary sodium. Journal of the American Public Health Association, 94(1), 19-22.

He, F. J., and MacGregor, G. (2003). How far should salt intake be reduced? American Heart Association, 42, 1093-1099.

Hebert, P. R., Bolt, R. J., Borhani, N. O., Cook, N. R. et al. (1995). Design of a multi-center trial to evaluate long-term life-style intervention in adults with high-normal blood pressure levels. Trials of hypertension prevention (phase II). Annals of Epidemiology, 5, 130-139.

J. Sainsbury plc. (2005). Corporate responsibility report: Salt reduction. United Kingdom. Retrieved June 1, 2006, from http://www.j-sainsbury.co.uk/files/reports/cr2005/index.asp?pageid=48

Joint National Committee on Prevention, Detection, Evaluation, and Treatment of High Blood Pressure. (1997). The Sixth Report of the Joint National Committee on Prevention, Detection, Evaluation, and Treatment of High Blood Pressure. Archives of Internal Medicine, 157, 2413-2446. 
(1988). The 1988 report of the Joint National Committee on Detection, Evaluation and Treatment of High Blood Pressure. Archives of Internal Medicine, 148, 1023-1038.

. (2003). The Seventh Report of the Joint National Committee on Prevention, Detection, Evaluation, and Treatment of High Blood Pressure: The JNC 7 report. Journal of the American Medical Association, 289, 2560-2572.

Kochanek, K. D., Smith, B. L., Anderson, R. (2001) Deaths: Preliminary data for 1999. National Vital Statistics Report, 49(3).

Lancaster, K. J. et al. (2004). Hypertension-related dietary patterns of rural older adults. Preventive Medicine, 38, 812-818.

Lasser, V. I., Raczynski, J. M., Stevens, V. J., Mattfeldt, M. K. et al. (1995). Trials of hypertension prevention, phase II. Structure and content of the weight loss and dietary sodium reduction interventions. Annals of Epidemiology, 5, 156-164.

Liu, K., and Stamler, J. (1984). Assessment of sodium intake in epidemiological studies. Annals of Clinical Research, 16, 49-54.

Loria, C. M., Obarzanek, E., and Ernst, N. D. (2001). Choose and prepare foods with less salt: Dietary advice for all Americans. The Journal of Nutrition, 131(suppl.), 536S-551S.

Manton, K. G., and Stallard, E. (1997). Health and disability differences among racial and ethnic groups. In L. G. Martin and B. J. Soldo (Eds.), Racial and ethnic differences in the health of older Americans. Washington, DC: National Academy Press.

Mattes, R. D., and Donnelly, D. (1991). Relative contributions of dietary sodium sources. Journal of the American College of Nutrition, 10, 383-93.

Panel on Dietary Reference Intakes for Electrolytes and Water, Standing Committee on the Scientific Evaluation of Dietary Reference Intakes, Food and Nutrition Board. (2004). Dietary reference intakes for water, sodium, potassium, chloride, and sulfate. Washington, DC: The National Academies Press.

Sacks, F. M., Svetkey, L. P., Vollmer, W. M. et al. (2001). Effects on blood pressure of reduced dietary sodium and the Dietary Approaches to Stop Hypertension (DASH) Diet. New England Journal of Medicine, 344, 3-10.

Schachter, J., Harper, P. H., Raden, M. E., Caggiula, A. W., McDonald, R. H., and Diven, W. F. (1980). Comparison of sodium and potassium intake with excretion. Hypertension, 2, 695-699.

Sanchez-Castillo, C. P., Branch, W. J., and James, W. P. (1987). A test of the validity of the lithiummarker technique for monitoring dietary sources of salt in men. Clinical Science (London), 72, 87-94.

SHEP Cooperative Research Group. (1991). Prevention of stroke by antihypertensive drug treatment in older persons with isolated systolic hypertension. Journal of the American Medical Association, 265, 3255-3264.

Svetkey, L. P., Harsha, D. W., Vollmer, W. M., Stevens, V. J. et al. (2003). Premier: A clinical trial of comprehensive lifestyle modification for blood pressure control: Rationale, design and baseline characteristics. Annals of Epidemiology, 13, 462-471.

Svetkey, L. P., Erlinger, T. P., Vollmer, W. M., Feldstein, A. et al. (2005). Effect of lifestyle modifications on blood pressure by race, sex, hypertension status, and age. Journal of Human Hypertension, 19, 21-31.

The Trials of Hypertension Prevention Collaborative Research Group. (1997). Effects of weight loss and sodium reduction intervention on blood pressure and hypertension incidence in overweight people with high-normal blood pressure. The trials of hypertension prevention, phase II. Archives of Internal Medicine, 157, 657-667.

U. S. Dept of Agriculture, Agricultural Research Service. (1996).Continuing survey of food intakes by individuals 1989-1991. Beltsville, MD: NTS Accession No. PB96-501747.

U. S. Dept of Agriculture, Agricultural Research Service. (2000). Continuing survey of food intakes by individuals 1994-1996. Beltsville, MD: NTS Accession No. PB2000-5000027. 
U. S. Department of Agriculture (2005). Dietary Guidelines Advisory Committee Report of the 2005 Dietary Guidelines for Americans. Washington, DC: Author.

U. S. Department of Health and Human Services, Centers for Disease Control and National Institutes of Health (2000). Heart disease and stroke. In US Department of Health and Human Services Healthy People 2010 Conference Edition (vol. 1), pp. 12-3-12-33. Washington, DC: Author.

Whelton, P. K. et al. (1997). Efficacy of nonpharmocologic interventions in adults with high-normal blood pressure: results from phase 1 of the trials of hypertension prevention. American Journal of Clinical Nutrition, 65(suppl.), 652S-660S.

Whelton, P. K., Appel, L. J., Espeland, M. A., Applegate, W. B. et al. (1998). Sodium reduction and weight loss in the treatment of hypertension in older persons. A randomized controlled trial of nonpharmacologic interventions in the elderly (TONE). Journal of the American Medical Association, 279, 839-846.

Whelton, P. K. et al. (2002). Primary prevention of hypertension: clinical and public health advisory from the national high blood pressure education program. Journal of the American Medical Association, 288, 1882-1888.

Whelton, P. K. et al. (2002)The dominance of salt in manufactured food in the sodium intake of affluent societies. Lancet, 1, 426-429.

World Health Organization. (2002). Reducing risks, promoting healthy life. World Health Report, 58. Geneva, Switzerland: Author.

Wright, J. et al. (2003). Dietary Intake of ten key nutrients for public health, United States: 1994-1996, 1999-2000. National Center for Health Statistics, Vital Health Statistics, Number 334.

Writing Group of the PREMIER Collaborative Research Group. (2003). Effects of comprehensive lifestyle modification on blood pressure control. Main results of the PREMIER clinical trial. Journal of the American Medical Association, 289, 2083-2093.

Zhou, B. F. et al. (2003). Nutrient intakes of middle-aged men and women in China, Japan, United Kingdom, and Unites States in the late 1990's: The INTERMAP Study. Journal of Human Hypertension, 17, 623-630.

\section{Acknowledgements}

A special thank you to the Center for Healthy Aging, University of Pittsburgh Graduate School of Public Health investigators and staff for their expertise and support. A special thanks to: N. Carole Milas, MS, RD, LDN (Essay Advisor) Department of Epidemiology, Graduate School of Public Health, University of Pittsburgh; Edmund M. Ricci, PhD (Committee Member), Department of Behavioral and Community Health Sciences, Graduate School of Public Health, University of Pittsburgh; Lewis H. Kuller, MD, DrPH (Committee Member), Department of Epidemiology, Graduate School of Public Health, University of Pittsburgh. The Center for Healthy Aging is funded by the Centers for Disease Control Cooperative Agreement U48/CCU320171 as a "Health Promotion and Disease Prevention Research Center."

Author Information

Courtney B. Johnson, MPH, RD

University of Hawaii at Manoa

Department of Public Health Sciences \& Epidemiology

John A. Burns School of Medicine

1960 East-West Road, Biomed D-210

Honolulu, HI 96822

Ph.: 808-956-5781

E-Mail: cbj@hawaii.edu 